\title{
Emotional Intelligence Moderates Anxiety Reactions in Chronic Health Conditions
}

\author{
Leehu Zysberg \\ The Graduate School, Gordon College of Education Haifa, Haifa, Israel
}

Email address:

leehuzysberg@yahoo.com

To cite this article:

Leehu Zysberg. Emotional Intelligence Moderates Anxiety Reactions in Chronic Health Conditions. American Journal of Applied

Psychology. Vol. 6, No. 3, 2017 pp. 38-41. doi: 10.11648/j.ajap.20170603.12

Received: June 26, 2017 Accepted: July 7, 2017 Published: August 1, 2017

\begin{abstract}
Anxiety associated with chronic health conditions is a serious comorbidity with implications for health promotion and quality of life in adults with chronic illness. Thus there is value in identifying personal resources that may protect against anxiety. A recent concept that may meet this definition is that of emotional intelligence (EI). It was hypothesized that EI moderates anxiety levels among individuals with chronic conditions. A sample of 268 women living in Israel was recruited for this preliminary investigation (208 without a chronic condition, 60 with a diagnosed chronic illness such as high blood pressure or diabetes). Participants completed validated measures of anxiety and EI and provided health and demographic information. The evidence supported the hypothesized moderating effect of EI on level of anxiety in individuals with and without chronic conditions. While a simple comparison showed that individuals with chronic conditions reported higher levels of anxiety than their peers without a chronic condition, EI and the interaction term of EI and health condition showed a significant effect on anxiety, nullifying the simple effect of health condition. This preliminary study supports the potential role of EI in the experience of anxiety among individuals with a chronic health condition. Should future research support these findings, screening for at-risk populations as well as future interventions may be developed to improve quality of life for individuals coping with chronic illness.
\end{abstract}

Keywords: Emotional Intelligence, Chronic Disease, Anxiety, Health, Cross-Sectional Design

\section{Background}

Chronic health conditions challenge individuals' daily function, may restrict abilities and activities, and present an ongoing threat to well-being and, at times, longevity $[1,2]$. Studies that focused on maintaining optimal well-being and quality of life in patients with chronic conditions have paid much attention to anxiety as a measurable concept representing ongoing distress that is not only a major challenge in its own right but is also identified as a major risk factor and even comorbidity that may exacerbate existing conditions $[3,4]$.

Researchers and practitioners are therefore interested in identifying risk factors as well as protective factors associated with anxiety and the challenges it poses in association with chronic disease. Although studies have identified social support, education, and socioeconomic status (SES) as potential protective factors in this context [5], there is less empirical evidence of individual-level resources that may protect against anxiety in individuals with chronic conditions.

This study examined one such potential factor, identified in the psychological and educational literature as a concept with much promise in this direction: emotional intelligence (EI). This relatively recent concept has been defined, either as an ability or as an amalgam of personality traits. Both definitions, however, share a common core: EI offers a conceptual framework for understanding how effectively individuals identify, process, and regulate emotions in themselves and others [6]. Measures of EI are associated with a broad range of life outcomes: from academic performance to job outcomes to, more recently, health outcomes [7, 8]. EI is hypothesized to have a protective effect on individuals facing health challenges through mechanisms related to stress processing and regulation [9].

If such is the case, can EI moderate the stress (and therefore, in the longer run, anxiety levels) evoked by chronic health conditions in individuals? Should evidence 
identify EI as a protective factor in this context, such understandings may be the basis for future screening for atrisk target populations and help practitioners develop intervention programs to reduce stress- and anxiety-related comorbidities in individuals with chronic health conditions.

It was therefore hypothesized that EI levels will moderate anxiety-level differences in populations with and without chronic health conditions.

\section{Method}

\subsection{Settings and Study Design}

A cross-sectional/correlational study design was adopted to test the hypothesized moderation model to minimize ethical issues and to get as close as possible to a target population that lives with chronic health conditions in the community (outside hospital and chronic care settings).

\subsection{Sample}

Two hundred sixty-eight women dwelling independently in their communities across Israel were recruited online, using snowball sampling, through a variety of social networks as part of a study of various health aspects of emotional experiences in daily life. Participants were divided into two groups based on their self-report of existing chronic conditions, which included diabetes, high blood pressure / coronary conditions, chronic pain, and cancer (in remission). Participants who reported an acute debilitating health condition $(n=4)$ were excluded from the sample. The chronic-condition subsample consisted of 60 participants, and the remaining participants reported not having any limiting chronic condition.

\subsection{Measures}

Participants completed a brief demographic questionnaire reporting their age, marital status, education, and income levels as well as the existence (and type) or absence of chronic illness. They also completed the following measures.

Emotional intelligence. EI was assessed using the Trait Emotional Intelligence Questionnaire Short Form [6], a 30item self-report measure of trait emotional intelligence, chosen for its internal stability, which typically ranges from.85 to.90, and its predictive validity in health-related studies [see: 7].

Anxiety. Anxiety was assessed using the Hamilton Anxiety Rating Scale, administered online as a self-directed, structured computerized interview. The 14-item scale is considered the gold standard in research and practice for assessing anxiety symptoms in many settings, including clinical ones (e.g., in depression). Internal reliability is acceptable at. 70 to. 74 . Known group validity was established in numerous studies [10].

\subsection{Procedure}

The procedure was approved by the author's IRB committee. Questionnaires were distributed online through social networks, and participants were asked to forward the link to the questionnaires to their acquaintances.

\subsection{Statistical Analyses}

Following the calculation of descriptive statistics and zeroorder correlations between the study variables, an analysis of covariance was conducted to compare participants with and without chronic conditions while controlling for background variables (age, SES) and testing the role of EI in this comparison. IBM-SPSS version 23.0 was used to perform these analyses.

\section{Results}

\subsection{Participant Characteristics}

Before the proposed moderation model was tested, sample statistics were obtained; these are summarized in Table 1.

Table 1. Sample descriptive statistics $(N=268)$.

\begin{tabular}{llll}
\hline Variable & Percentage & Mean (SD) & Min/Max \\
\hline Age (years) & - & $39.94(9.05)$ & $19 / 65$ \\
Education level & 1 & - & - \\
Elementary & 5 & - & - \\
High school & 5 & - & - \\
Post-secondary & 49 & - & - \\
BA or equivalent & 40 & - & - \\
MA or above & & - & - \\
Income level & & - & - \\
Much below national median & 4 & - & - \\
A little below median & 7 & - & - \\
Around the median & 20 & - & - \\
A little above median & 35 & - & - \\
Much above the median & 34 & - & - \\
Chronic health condition & & - & - \\
None & 77 & - & - \\
Yes* & 23 & - & - \\
Ethnic group & & - & - \\
Jewish & 88 & - & - \\
Christian & 5 & & - \\
Muslim & 7 & & - \\
\hline
\end{tabular}

* For reported conditions see text.

The statistics suggest a sample that belongs in the upper middle class in terms of education and income level. A relatively high percentage of participants reported having at least one officially diagnosed chronic condition. The conditions reported were diabetes, high blood pressure, chronic pain (of undiagnosed origin), cardiac conditions, and cancer (in remission).

\subsection{Hypothesis Testing}

EI and anxiety scores were converted to standardized $\mathrm{Z}$ scores in order to test the hypothesized model. The analysis was first run using only the health-conditions groups and demographics (education and income level) as independent variables and anxiety as the dependent variable. The results are summarized in Table 2. 
Table 2. Summary results of analysis of covariance comparing anxiety levels of participants with and without a chronic condition and controlling for demographic variables.

\begin{tabular}{llll}
\hline Variable & $\begin{array}{l}\text { Mean (SD) in the } \\
\text { group with no chronic } \\
\text { conditions }(\boldsymbol{n}=\mathbf{2 0 8})\end{array}$ & $\begin{array}{l}\text { Mean (SD) in the } \\
\text { group with chronic } \\
\text { conditions }(\boldsymbol{n}=\mathbf{6 0})\end{array}$ & $\boldsymbol{F}(\mathbf{d f})$ \\
\hline Anxiety & $.56(1.03)$ & $-.07(.98)$ & $10.42(1$, \\
& & $4.30(1.00)$ & $\mathrm{NS}$ \\
Education & $4.33(1.08)$ & $3.95(1.09)$ & $\mathrm{NS}$ \\
Income & $3.90(1.07)$ & & \\
\hline
\end{tabular}

${ }^{*} \mathrm{p}<.05 * * \mathrm{p}<.01$

The results lend support to the existing literature: participants with chronic health conditions reported higher levels of anxiety. Demographic variables did not significantly interfere with this effect. In the second step, a linear multiple regression was calculated with condition, EI, and the interaction term inserted while controlling for demographics. The results are displayed in Table 3.

Table 3. Summary results of multiple regression for anxiety levels using condition group, emotional intelligence, and an interaction term as independent variables $(N=268)$.

\begin{tabular}{lll}
\hline Variable & Beta & Zero-order Pearson's $\boldsymbol{r}$ \\
\hline Group (with and without chronic & -.12 & $-.16^{*}$ \\
conditions) & $-.32^{* *}$ & $-.39^{* *}$ \\
EI & $-16^{* *}$ & $-.25^{* *}$ \\
EI $\times$ Group & -.08 & -.12 \\
Age & -.09 & $-.17^{*}$ \\
Income & .01 & -.10 \\
Education & & \\
\hline
\end{tabular}

$* \mathrm{p}<.05 * * \mathrm{p}<.01$

The analysis results support the hypothesized model: the group differences disappeared, whereas both EI and the interaction term showed strong and medium effect sizes, respectively. Demographic variables (age, income, and education level) did not show significant effects when inserted alongside the main independent variables.

\section{Discussion}

The psychosocial toll of chronic illness is well documented in the literature [11]. Although interventions are being developed and better care protocols tested, it may also be of value to identify personal resources that can buffer the effects of chronic conditions and ameliorate stress, anxiety, and their secondary effects on individuals and their social circles. EI emerges from the recent literature as a potential resource with such properties: it has been demonstrated to buffer the effects of stress and to show beneficial effects for chronic conditions such as diabetes [8, 12]. Can EI serve as a protective factor against anxiety in chronically ill individuals? This study put this question to a preliminary test in a snowball sample of upper-middle-class women living and working in Israel.
The results support the hypothesized role of EI: whereas simple comparisons showed higher levels of reported anxiety among participants with chronic conditions than among those with no chronic condition, this effect disappeared when EI and the interaction between EI and health-condition group were added to the analyses. EI showed a strong effect on anxiety levels, and the interaction term showed an additional medium-size effect. Interestingly, demographics did not play a significant role and did not interfere with the effects reported here. These findings fit the existing theoretical and empirical literature on EI, suggesting that this concept represents individuals' ability and tendency to 'work well with emotions' and to regulate emotional responses in a manner that allows more efficient coping $[9,13]$. The results may suggest that individuals with chronic conditions indeed cope with higher levels of anxiety. However, they also suggest that EI plays a major role in determining the experience of anxiety in general (even when unrelated to the chronic condition) and moderates anxiety among those in the chronic-condition group.

Although these conclusions are encouraging, readers should consider the study limitations in interpreting the results. The sample was modest in size, included women only, and was biased culturally and socioeconomically. However, these apparent limitations may actually lend more validity to the results: Israel provides full health coverage to every citizen or resident (which may be one reason why we did not find that income affected our results); and Israeli culture is a blend of mostly Western and Mediterranean values, norms, and behavior patterns [14] and may therefore reflect more cultural diversity than other samples might. In addition, finding these results in a relatively 'robust' sample of uppermiddle-class women (who may have better access to resources than lower-SES individuals) suggests that in lowerincome, lower-education samples such results may be even more dramatic - as these populations are usually considered more vulnerable [15]. The use of self-report measures (although showing good reliability and validity indices) may lead to additional biases, and calls for future studies to use more 'objective' measures, especially as outcomes.

\section{Conclusion}

These limitations notwithstanding, the evidence provides preliminary directions for future investigation: the potential of EI as a protective factor among people with chronic illness should be addressed in broader samples, including diverse target population segments, and under more conditions. Use of a broader range of measures, especially for outcome assessment, may help support and further validate the direction pointed to in this study. Should further evidence support the findings reported herein, such knowledge may be a basis for future screening for high-risk individuals and groups as well as for developing interventions to support individuals coping with the consequences of their chronic conditions. 


\section{References}

[1] Brenes GA. Anxiety and chronic obstructive pulmonary disease: prevalence, impact, and treatment. Psychosom Med. 2003; 65(6):963-70. doi: 10.1097/01. PSY.0000097339.75789.81.

[2] Pereira BDS, Fernandes NdS, de Melo NP, Abrita R, Grincenkov FRDS, Fernandes NMdS. Beyond quality of life: a cross sectional study on the mental health of patients with chronic kidney disease undergoing dialysis and their caregivers. Health Qual Life Outcomes. 2017; 15(1):74. doi: 10.1186/s12955-017-0646-4.

[3] Kepka S, Baumann C, Anota A, Buron G, Spitz E, Auquier P, Guillemin F, Mercier M. The relationship between traits optimism and anxiety and health-related quality of life in patients hospitalized for chronic diseases: data from the SATISQOL study. Health Qual Life Outcomes. 2013; 11(1):134. doi: 10.1186/1477-7525-11-134.

[4] Yohannes AM, Willgoss TG, Baldwin RC, Connolly MJ. Depression and anxiety in chronic heart failure and chronic obstructive pulmonary disease: prevalence, relevance, clinical implications and management principles. Int $\mathrm{J}$ Geriatr Psychiatry. 2010; 25(12):1209-21. doi: 10.1002/gps.2463.

[5] Broadhead WE, Kaplan BH, James SA, Wagner EH, Schoenbach VJ, Grimson R, Heyden S, Tibblin G, Gehlbach $\mathrm{SH}$. The epidemiologic evidence for a relationship between social support and health. Am J Epidemiol. 1983; 117(5):52137. doi: 10.1093/oxfordjournals.aje.a113575.

[6] Petrides KV. Ability and trait emotional intelligence. In: Chamorro-Premuzic T, von Stumm S, Furnham A, editors. The Wiley-Blackwell handbook of individual differences. Malden, MA: Wiley-Blackwell; 2011: 656-78 doi: 10.1002/9781444343120.ch25.

[7] Schutte NS, Malouff JM, Thorsteinsson EB, Bhullar N, Rooke
SE. A meta-analytic investigation of the relationship between emotional intelligence and health. Pers Individ Dif. 2007; 42(6):921-33. doi: 10.1016/j.paid.2006.09.003.

[8] Zysberg L, Bar Yosel T, Goldman M. Emotional intelligence and glycemic management among type I diabetes patients. J Health Psychol. 2015; 22(2):158-63. doi: 1359105315596373.

[9] Zysberg L. Emotional intelligence and health outcomes: toward an ecological model of well-being. New York: Novinka; 2016.

[10] Maier W, Buller R, Philipp M, Heuser I. The Hamilton Anxiety Scale: reliability, validity and sensitivity to change in anxiety and depressive disorders. J Affect Disord. 1988; 14(1):61-8. doi: 10.1016/0165-0327(88)90072-9.

[11] Martire LM, Lustig AP, Schulz R, Miller GE, Helgeson VS. Is it beneficial to involve a family member? A meta-analysis of psychosocial interventions for chronic illness. Health Psychol. 2004; 23(6):599-611. doi: 10.1037/0278-6133.23.6.599.

[12] Augusto Landa JM, Lopez-Zafra E, Berrios Martos MP, Aguilar-Luzon MDC. The relationship between emotional intelligence, occupational stress and health in nurses: a questionnaire survey. Int J Nurs Stud. 2008; 45(6):888-901. doi: 10.1016/j.ijnurstu.2007.03.005.

[13] Saklofske DH, Austin EJ, Galloway J, Davidson K. Individual difference correlates of health-related behaviours: preliminary evidence for links between emotional intelligence and coping. Pers Individ Dif. 2007; 42(3):491-502. doi: 10.1016/j.paid.2006.08.006.

[14] Pines AM, Zaidman N. Gender, culture, and social support: a male-female, Israeli Jewish-Arab comparison. Sex Roles. 2003; 49(11):571-86. doi: 10.1023/B:SERS.0000003128.99279.94.

[15] López-Calva LF, Ortiz-Juarez E. A vulnerability approach to the definition of the middle class. J Econ Inequal. 2014; 12(1): 23-47. doi: 10.1007/s10888-012-9240-5. 